\title{
Unitarity constraint on the Kähler curvature
}

\author{
Yohei Ema, ${ }^{a}$ Ryuichiro Kitano ${ }^{a, b}$ and Takahiro Terada ${ }^{a}$ \\ ${ }^{a}$ Theory Center, KEK, \\ 1-1 Oho, Tsukuba, Japan \\ ${ }^{b}$ Department of Particle and Nuclear Physics, \\ Graduate University for Advanced Studies (Sokendai), \\ 1-1 Oho, Tsukuba, Japan \\ E-mail: ema.yohei@gmail.com, ryuichiro.kitano@kek.jp, \\ teradat@post.kek.jp
}

ABSTRACT: In supersymmetric theories, the signs of quartic terms in the Kähler potential control the stability of non-supersymmetric field configurations. In particular, in supersymmetric inflation models, the signs are important for the stability of an inflationary trajectory as well as for the prediction of the spectral index. In this paper, we clarify what properties of a UV theory determine the sign from unitarity arguments of scattering amplitudes. As non-trivial examples, we discuss the sign of a four-meson term in large $N$ supersymmetric gauge theories and also those of the quartic terms obtained in the intersecting D-brane models in superstring theory. The UV origins of inflationary models and supersymmetry breaking models are constrained by this discussion.

KeYwords: Scattering Amplitudes, Supersymmetric Effective Theories, 1/N Expansion, Compactification and String Models

ARXIV EPRINT: 1807.06940 


\section{Contents}

1 Introduction 1

2 Phenomenological motivations 2

3 UV theories and their effective theories 4

4 Amplitude analysis $\quad 5$

$5 \quad$ Large $N$ supersymmetric QCD $\quad 6$

$\begin{array}{lll}6 & \text { String theory amplitudes } & 9\end{array}$

$\begin{array}{lll}7 \text { Conclusion } & 10\end{array}$

\section{Introduction}

Effective theories are quite strong tools to study low energy physics especially when the UV theories are strongly coupled or even unknown, but they usually involve an infinite number of parameters, even after requiring the symmetry properties. The analyticity and unitarity of the scattering amplitudes provide a set of non-trivial (in)equalities for the parameters in the effective theories [1-6]. After the recent revival of this discussion by ref. [4], various non-trivial results have been obtained. Examples include the UV inconsistency of the brane gravity model (the DGP model [7]) [4], the bound on the value of the superpotential in supersymmetric theories [8], and the proof of the $a$-theorem in four dimensions [9].

In $\mathcal{N}=1$ supersymmetric theories, while the superpotential in the low energy effective theory is strongly constrained by holomorphy, the Kähler potential is usually not determined. The quartic terms in the Kähler potential are often important as they modify the shape of the scalar potential. For example, in the O'Raifeartaigh models of supersymmetry breaking [10], the pseudo-modulus field, which is the scalar component of the supersymmetry breaking chiral superfield, has no potential at the classical level, and the quartic Kähler term generated at the quantum level stabilizes or destabilizes the vacuum depending on the sign of the coefficient. (See, e.g., refs. [11-13] for discussions of some general features of the O'Raifeataigh models.)

The importance of the quartic terms is not limited to the vacuum of the theory. They are relevant whenever supersymmetry is broken, particularly during inflation. This is because the quartic terms generate supersymmetry-breaking mass terms (Hubble-induced mass terms) of scalar fields in the theory through the coupling to the field whose $F$ term drives inflation. They affect the curvature along and/or orthogonal to the inflaton trajectory, and hence the spectral index and/or the stability of the trajectory. 
In this paper, we study constraints on the sign of the quartic Kähler term from the unitarity arguments of the scattering amplitudes. We first explain phenomenological motivations in section 2 . Then we compare the effective field theory having the quartic Kähler term with its several UV completions in section 3. Unlike the $\mathcal{O}\left(p^{4}\right)$ terms in the chiral Lagrangian, there are no universal predictions on the sign of the coefficients [14-16]. The amplitude analysis in section 4, however, makes it clear which UV information determines the sign. In section 5, as a non-trivial case with the strong coupling, we examine the supersymmetric QCD at large $N$ and find that the sign is fixed in the leading order of the $1 / N$ expansion. Since the open-string-like features of the large $N$ expansion play crucial roles, we expect that the same sign is realized in a wide universality class of UV completions, such as the D-brane models in string theory. This is discussed in section 6. Finally, section 7 is devoted to a summary and discussion.

\section{Phenomenological motivations}

The quartic term in the Kähler potential modifies the shape of the scalar potential when supersymmetry is spontaneously broken. Thus its sign is phenomenologically important, e.g., for the stability of the supersymmetry breaking vacuum or for the inflationary dynamics.

To be specific, we consider the model with the following Kähler potential:

$$
K=X^{\dagger} X-\frac{c}{4 \Lambda^{2}}\left(X^{\dagger} X\right)^{2}+\cdots
$$

where $X$ is a chiral superfield, $c$ is a dimensionless constant, and $\Lambda$ is the UV cutoff scale. This form of the Kähler potential is general when $X$ is charged under some (global) symmetry, which is the case for all the examples we consider in this paper. Note that $R$-symmetry is a good symmetry in a wide class of supersymmetry breaking models [17].

The sign of $c$ is important, for instance, when $X$ has a linear superpotential term, $W=m^{2} X$. In this case, the model describes the $F$-term supersymmetry breaking by $F_{X}=m^{2}$. When $c>0$, the minimum is stabilized at $X=0$, otherwise one cannot establish the presence of the vacuum. If eq. (2.1) is a low-energy effective theory of some UV physics, such as strongly coupled gauge theories or string theories, it is a non-trivial question whether one can obtain $c>0$ as desired. An example is $\mathrm{SU}(N)$ supersymmetric QCD with $N_{f}=N$ flavors of light quarks discussed in ref. [18], where $c>0$ is anticipated in ref. [18] while no evidence has been provided [19].

In the context of inflation, the sign of the quartic Kähler term is important when it contains fields whose $F$-terms drive inflation, since such a term generates Hubble induced mass terms. It controls the curvature of the scalar manifold in two ways: (i) the curvature along the inflaton trajectory, which is related to the spectral index $n_{\mathrm{s}}$, and (ii) the stability of the orthogonal directions to the inflaton trajectory, are affected. When the trajectory has a bend, the quartic term is related also to the sound speed and hence non-Gaussianity [20].

For instance, the supersymmetric hybrid inflation in its original version [21] predicts $n_{\mathrm{s}} \gtrsim 0.98$ which is in tension with the latest Planck observation [22]. Once we introduce a 
quartic Kähler term, however, the model

$$
\begin{aligned}
K & =X^{\dagger} X+\phi^{\dagger} \phi+\bar{\phi}^{\dagger} \bar{\phi}-\frac{c}{4 \Lambda^{2}}\left(X^{\dagger} X\right)^{2}+\ldots, \\
W & =\kappa X\left(\phi \bar{\phi}-\mu^{2}\right)
\end{aligned}
$$

gives a lower value of $n_{\mathrm{s}}$ for a negative $c$ [23], while a positive $c$ makes the fit worse, where $X$ is a gauge singlet (inflaton), and $\phi(\bar{\phi})$ is a (anti-)fundamental representation of $\mathrm{SU}(N)$ (waterfall field). A similar situation occurs for supersymmetric new (hilltop) inflation $[24,25]$, as the model

$$
\begin{aligned}
& K=X^{\dagger} X-\frac{c}{4 \Lambda^{2}}\left(X^{\dagger} X\right)^{2}, \\
& W=v^{2} X-\frac{g}{n+1} X^{n+1},
\end{aligned}
$$

with a small negative $c$ improves the fit to the Planck result (unless $n$ is too large) [26].

As an example of the case (ii), let us consider a chaotic inflation model with a shift symmetry in Kähler potential [27]:

$$
\begin{aligned}
K & =\frac{1}{2}\left(\phi+\phi^{\dagger}\right)^{2}+X^{\dagger} X-\frac{c}{4 \Lambda^{2}}\left(X^{\dagger} X\right)^{2}+\ldots \\
W & =X f(\phi)
\end{aligned}
$$

where (the imaginary part of) $\phi$ is the inflaton and $X$ is the so-called stabilizer field whose $F$-term drives inflation. In this class of models, $c$ must be non-negative to ensure the positive mass squared of $X$ during inflation with a generic initial condition. In models without the stabilizer field [28-30], the shift-symmetric inflaton potential becomes unbounded from below by the supergravity term $-3 e^{K}|W|^{2}[27,30]$. It is cured by introducing the shiftsymmetric quartic term [31-34], which also requires $c>0$.

More generally, we can consider couplings $-\frac{c_{\psi}}{\Lambda^{2}} X^{\dagger} X \psi^{\dagger} \psi$ in the Kähler potential where $X$ is the field whose $F$-term drives inflation and $\psi$ is some other field. If $c_{\psi}$ is positive (and sizable), the scalar components of $\psi$ obtain a positive Hubble-induced mass squared so that they tend to be stable during inflation without generating any isocurvature perturbations.

Finally, we comment on a class of inflation models utilizing the Kähler potential of the form $K=-3 \alpha \log \left(\Phi+\Phi^{\dagger}\right)$, such as $\alpha$-attractor models [35-37]. The quartic Kähler term is related to the curvature of the Kähler manifold as $R=c / \Lambda^{2}$ at the origin, and the Kähler curvature is negative in $\alpha$-attractor models of inflation, $R=-2 /(3 \alpha)<0 .{ }^{1}$ Similarly, the Kähler curvature is negative for inflationary attractors other than the $\alpha$ attractor [38]. The role of the quartic Kähler term has been discussed in ref. [39] in the context of modular inflation.

\footnotetext{
${ }^{1}$ Indeed, we can rewrite the Kähler potential as $K=-3 \alpha \log \left(1-X^{\dagger} X\right)$ under the field redefinition $\Phi=(1+X) /(1-X)$ up to a Kähler transformation. In this basis, after expanding it around the origin and taking the canonical normalization, it is transparent that the Kähler curvature is determined by the quartic term.
} 


\section{UV theories and their effective theories}

Before the discussion of the scattering amplitudes, let us consider some examples that reduce to the model in eq. (2.1). The first model we consider is

$$
\begin{aligned}
K & =X^{\dagger} X+Y^{\dagger} Y+Z^{\dagger} Z, \\
W & =\frac{\lambda}{2} X^{2} Y+m Y Z
\end{aligned}
$$

By integrating out the $Y$ and $Z$ field at tree level, one obtains

$$
\begin{aligned}
& K_{\text {eff }}=X^{\dagger} X+\frac{|\lambda|^{2}}{4 m^{2}}\left(X^{\dagger} X\right)^{2}+\cdots, \\
& W_{\text {eff }}=0 .
\end{aligned}
$$

We obtain $c<0$.

The second model we consider is the same Kähler potential but with

$$
W=\frac{\lambda}{2} X Y^{2}+m Y Z .
$$

This is the O'Raifeartaigh model when we add the linear term of $X$ in the superpotential. It is known that the model has a minimum at $X=0$ by evaluating one-loop effective potentials. Indeed, by integrating out $Y$ and $Z$ at the one-loop level, the effective theory is given by

$$
\begin{aligned}
& K_{\text {eff }}=X^{\dagger} X-\frac{|\lambda|^{4}}{64 \pi^{2} m^{2}}\left(X^{\dagger} X\right)^{2}+\cdots \\
& W_{\text {eff }}=0
\end{aligned}
$$

One finds $c>0$.

We have already seen that there is no universal constraint on the sign of $c$. There is also an example to give $c>0$ at tree level:

$$
K=X^{\dagger} e^{-2 V} X+m^{2} V^{2},
$$

where $V$ is a massive vector superfield which has the kinetic term,

$$
f=\frac{1}{4 g^{2}} W^{\alpha} W_{\alpha} .
$$

By integrating out $V$, one obtains the effective theory:

$$
\begin{aligned}
& K_{\text {eff }}=X^{\dagger} X-\frac{g^{2}}{m^{2}}\left(X^{\dagger} X\right)^{2}+\cdots, \\
& W_{\text {eff }}=0 .
\end{aligned}
$$

In the following section, we examine which UV information determines the sign of $c$ by means of the analysis of the scattering amplitude. 


\section{Amplitude analysis}

We consider the scattering amplitude of the scalar component of $X$. The amplitude of $X X^{\dagger} \rightarrow X X^{\dagger}$ at a low energy is given by

$$
A_{X X^{\dagger}}(s, t)=\frac{c}{\Lambda^{2}}(s+t)+\cdots .
$$

The coefficient of the leading term is matched to the one in the effective theory in eq. (2.1), and "..." represents higher order terms in the energy expansions. Since we are interested in the sign of the contact four-point term obtained by integrating out the UV modes rather than in the loop contributions of light modes, the latter contribution (if any) is subtracted in eq. (4.1).

Following the discussion in ref. [4], we perform a contour integral,

$$
\frac{1}{2 \pi i} \int_{C} d s \frac{A_{X X^{\dagger}}(s, 0)}{s^{2}},
$$

in figure 1. There is a simple pole at $s=0$ due to eq. (4.1). The cuts along the positive and negative real axes represent the on-shell intermediate states in the $s$ - and $u$-channels, respectively. If $X$ itself and/or other massless particles contribute to the intermediate states, one should introduce IR regulators in eq. (4.2) so that the contour enclosing the pole can evade the cuts. This IR deformation does not modify the conclusions [4].

The discontinuity across the cut and/or pole for $s>0$ is given by

$$
\left.\operatorname{Disc}\left[A_{X X^{\dagger}}(s, 0)\right]\right|_{s>0}=2 i s \sigma_{X X^{\dagger}}(s),
$$

by the optical theorem, where $\sigma_{X X^{\dagger}}(s)$ is the total cross section of $X X^{\dagger} \rightarrow$ anything, which is positive. By the crossing symmetry, one can also obtain,

$$
\left.\operatorname{Disc}\left[A_{X X^{\dagger}}(s, 0)\right]\right|_{s<0}=2 i s \sigma_{X X}(-s),
$$

where $\sigma_{X X}(s)$ is the total cross section of $X X \rightarrow$ anything.

When the large $|s|$ part does not contribute to the integral, one obtains a relation,

$$
\frac{c}{\Lambda^{2}}=\frac{1}{\pi} \int_{0}^{\infty} d s\left(\frac{\sigma_{X X^{\dagger}}(s)}{s}-\frac{\sigma_{X X}(s)}{s}\right) .
$$

One can see that $c$ can have both signs in principle. Note that the sign is determined by the relative sizes between the total cross sections of $X X^{\dagger}$ and $X X$ channels. A similar discussion for four-fermion operators has been given in ref. [6].

By looking at the examples in the previous section, one can confirm the above relation. In the first example [eqs. (3.1) and (3.2)], the $X X \rightarrow Z^{\dagger}$ process is possible at the leading order in $\lambda$ while the amplitude for the $X X^{\dagger} \rightarrow Y Y^{\dagger}$ process is $\mathcal{O}\left(\lambda^{2}\right)$, hence $c<0$. In the second example [eqs. (3.1) and (3.5)], only the $X X^{\dagger} \rightarrow Y Y^{\dagger}$ or $Z Z^{\dagger}$ processes are possible at tree level, leading to $c>0$.

The third example [eqs. (3.8) and (3.9)] is a bit tricky. It looks the sign, $c>0$, is consistent with the fact that only the $X X^{\dagger} \rightarrow V$ contribution is non-vanishing at $\mathcal{O}(g)$, 


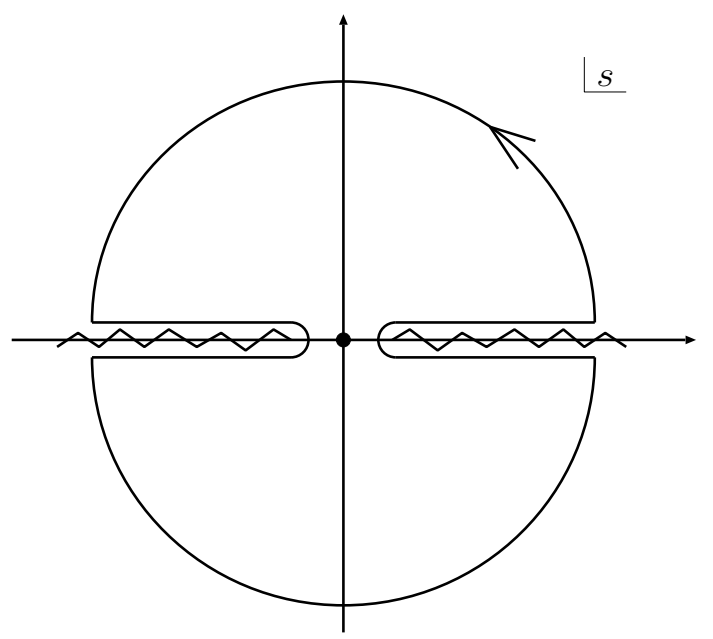

Figure 1. Contour $C$ of the integral.

but actually the large $|s|$ part of the integral does not vanish. The contribution from the $t$-channel exchange of $V$ grows linearly in $s .^{2}$ In this case, we cannot use eq. (4.5) for the determination of the sign of $c$.

The relation in eq. (4.5) helps us to find out the sign of $c$ without computing the Feynman diagrams. In generic situations, one finds $c>0$. The processes $X X^{\dagger} \rightarrow$ anything are typically possible at tree level, whereas $X X \rightarrow$ anything requires an appropriate final state and interactions to be present. To conclude it, however, one has to ensure that the large $|s|$ part of the integral is negligible.

\section{$5 \quad$ Large $N$ supersymmetric QCD}

In the examples so far, we can readily derive the quartic term after integrating out heavy degrees of freedom in the UV models. In this section, we consider a more non-trivial example: the large $N$ supersymmetric QCD with $N_{f}$ flavors of light quarks. In supersymmetric gauge theories, the low energy physics is often described by gauge singlet chiral superfields such as mesons, $M$ [40]. The Kähler potential for those fields is by assumption smooth at the origin, and thus can be expanded around it. For a large $N$, the mesons are weakly coupled, and the scattering amplitudes are assumed to be systematically expanded in powers of $1 / N$.

Our main interest is in the situation where effective superpotential is non-singular at the origin, such as the cases with the quantum deformed moduli space or the s-confinement. That requires $N_{f} \geq N$ for the supersymmetric QCD [40]. In such a situation, it is more suitable to consider the topological expansion: taking large $N$ while $N_{f} / N$ fixed [41]. The s-confinement with a fixed number of $N_{f}$ is possible once we extend the theory, e.g. by including antisymmetric tensor fields [42] which are treated as double lines in the $1 / N$ expansion. We here assume that the $1 / N$ expansion or the topological expansion provides

\footnotetext{
${ }^{2}$ This is barely consistent with perturbative unitarity. The partial wave amplitudes grow logarithmically corresponding to the fact that perturbation finally breaks down when logarithms become large.
} 


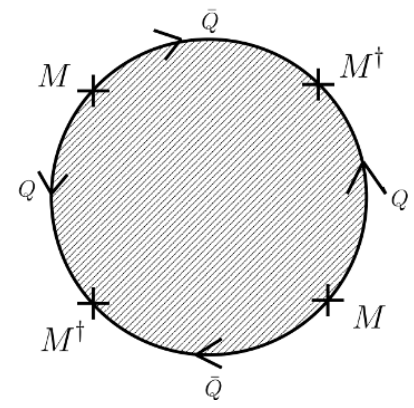

Figure 2. The leading diagram for the meson-meson scattering.

a qualitatively correct picture for the scattering amplitudes of the mesons in the background of $M=0$. Under the assumption, we will see below that $c>0$ at the leading order.

At the leading order of the $1 / N$ expansion, the low energy effective theory in the meson sector is given by

$$
K=\operatorname{Tr}\left(M^{\dagger} M\right)-\frac{c}{4 \Lambda^{2}} \operatorname{Tr}\left(M^{\dagger} M M^{\dagger} M\right),
$$

where $c$ is $\mathcal{O}(1 / N)$.

Below, we consider the scattering amplitudes among mesons. To this end, we decompose the $N_{f} \times N_{f}$ meson matrix as

$$
M=\sqrt{2} M^{a} T^{a}+\frac{1}{\sqrt{N}_{f}} M^{0} \cdot \mathbf{1}, \quad\left(a=1, \cdots, N_{f}^{2}-1\right)
$$

where $T^{a}$ are the generators of $\mathrm{SU}\left(N_{f}\right)$, so that $M^{a}$ and $M^{0}$ are canonically normalized. For example, the scattering amplitude of $M^{a} M^{0 \dagger} \rightarrow M^{a} M^{0 \dagger}$ at low energy is

$$
A_{M M^{\dagger}}^{(a 0)}(s, t)=\frac{c}{\Lambda^{2}}(s+t)+\cdots
$$

The amplitude at this order is given by the diagram in figure 2. Here, the arrows represent the flow of chirality, not that of color. The imaginary part of the forward amplitude, $A_{M M^{\dagger}}^{(A B)}(s, 0),\left(A, B=0,1, \cdots, N_{f}^{2}-1\right)$, for $s>0$ is the total cross section of $M^{A} M^{B^{\dagger}} \rightarrow$ anything.

In the case where $N_{f} / N$ is fixed, the final states of the $M^{A} M^{B \dagger}$ channel include resonances at $\mathcal{O}(1 / N)$ as well as $n$-meson states at $\mathcal{O}\left(N_{f}^{n-1} / N^{n}\right)$. Therefore, there is a cut in the real positive axis in the amplitude. For fixed $N_{f}$, there are only poles of the resonances.

On the other hand, the final states of the $M^{A} M^{B}$ channel do not have a resonance at $\mathcal{O}(1 / N)$. The chirality flow does not allow us to have three-point vertices. The multi-meson final states are also suppressed by the same reason.

Therefore, at $\mathcal{O}(1 / N)$, the discontinuities of the amplitudes are only on the $s>0$ axis. By unitarity, they contribute positively to $c$ as we have seen. If the large $|s|$ part of the integral vanishes, one can conclude $c>0$. One can separately discuss the large $|s|$ behaviors for the cases of fixed $N_{f} / N$ and fixed $N_{f}$. 
With $N_{f} / N$ fixed, the total cross section is dominated by the multi-particle final states. This is the same situation as the hadron scatterings in the real QCD where the high-energy scattering is dominated by the inelastic scattering, i.e., by the imaginary part, $\operatorname{Re} A_{M M^{\dagger}}(s, 0) \ll \operatorname{Im} A_{M M^{\dagger}}(s, 0)$. In such situations, the difference between $\operatorname{Im} A_{M M^{\dagger}}(s, 0) / s$ and $\operatorname{Im} A_{M M}(s, 0) / s$ vanishes at a large $s$ by Pomeranchuk's theorem $[43,44]$. Since $\operatorname{Im} A_{M^{\dagger} M}(-s, 0)=\operatorname{Im} A_{M M}(s, 0)=0$ for $s \rightarrow \infty, A_{M M^{\dagger}}(s, 0) / s \rightarrow 0$ for $s \rightarrow \infty$, which implies that the large $|s|$ part of the integral vanishes. Therefore, we can conclude $c>0$ at $\mathcal{O}(1 / N)$.

With $N_{f}$ fixed, we may first define $\hat{c}(t)$ as

$$
\left.\frac{\hat{c}(t)}{\Lambda^{2}} \equiv \frac{d}{d s} A_{M M^{\dagger}}^{(a 0)}(s, t)\right|_{s=0}=\frac{1}{2 \pi i} \int_{C} d s \frac{A_{M M^{\dagger}}^{(a 0)}(s, t)}{s^{2}},
$$

where the original $c$ is given by $c=\hat{c}(0)$. There are only poles at the leading order in $1 / N$. The locations of the $s$ poles of $A_{M M^{\dagger}}^{(a 0)}(s, t)$ are independent of $t$, and vice versa, since there is no $u$-channel process at this order. Thus $A_{M M^{\dagger}}^{(a 0)}(s, t)$ for a fixed $s$ has poles only in the $t>0$ region, as there are no tachyons nor massless particles in the $t$-channel. This indicates that $\hat{c}(t)$ also has singularities only in the $t>0$ region as one can see from the second expression.

The integral can be decomposed, as usual, to the large $|s|$ part and the discontinuity part expressed by the imaginary part of the amplitude. By writing the large $|s|$ behavior as the Regge trajectory, $A(s, t) \sim s^{j(t)}$, it has recently been found that there is a universal behavior, $j(t) \sim \alpha^{\prime} t+\cdots$, in the large positive $t$ [45]. For a large negative $t$, which corresponds to the fixed angle scatterings at high energy, the amplitude should behave according to the quark counting rule, $j(t) \sim-2[46,47]$. Therefore, by taking $t$ at a sufficiently negative value, the large $|s|$ part does not contribute. See refs. [48] and [49] for explicit constructions of amplitudes to realize the asymptotic behaviors at positive and negative $t$.

The function $\hat{c}(t)$ around $t=t_{0}$ for a large negative $t_{0}$ is expressed as

$$
\frac{\hat{c}(t)}{\Lambda^{2}}=\frac{1}{\pi} \int_{0}^{\infty} d s \frac{\operatorname{Im} A(s, t)}{s^{2}}=\sum_{k=0}^{\infty} c_{k}\left(t_{0}\right)\left(t-t_{0}\right)^{k},
$$

where

$$
c_{k}\left(t_{0}\right)=\frac{1}{\pi} \int_{0}^{\infty} d s \frac{a_{k}\left(s, t_{0}\right)}{s^{2}}
$$

and

$$
\operatorname{Im} A(s, t)=\sum_{k=0}^{\infty} a_{k}\left(s, t_{0}\right)\left(t-t_{0}\right)^{k} .
$$

The behavior at a large negative $t$ ensures that the integrals converge, and the expansion around $t=t_{0}$ in eq. (5.5) is defined. Since the singularities of $\hat{c}(t)$ are only in the $t>0$ region, the radius of convergence is larger than $\left|t_{0}\right|$. In particular, the $t \rightarrow 0$ limit of the integral in eq. (5.5) is valid, and by $\operatorname{Im} A(s, 0)>0$, we conclude $c>0$. 
We stress that the absence of $u$-channel poles is crucial in this discussion. If there were $s$ and $u$-channel poles simultaneously, $\hat{c}(t)$ generally would have poles in the region $t<0$ since the locations of the $u$-channel poles are $t$-dependent. In that case, the radius of convergence could be smaller than $\left|t_{0}\right|$. If $A_{X X^{\dagger}}$ has only $u$-channel discontinuities, we can instead consider $A_{X X}$ which is related to $A_{X X^{\dagger}}$ by the $s-u$ crossing.

The above discussion based on the behavior of the fixed angle scatterings and the location of the singularities of $t$ is not limited to the case where the amplitudes have only simple poles. For example, the same discussion could have applied to the case of fixed $N_{f} / N$.

The high-energy behavior of the forward amplitude implies that the Froissart bound [50], $A(s, 0) \lesssim s \log ^{2} s$, is not saturated in both cases. On the other hand, in the real world QCD, the growth of the total cross section, $\sigma(s)=\operatorname{Im} A(s, 0) / s$, is experimentally observed. This may be explained by contributions from higher order terms in $1 / N$, such as the exchange of the Pomeron. (See, e.g., [51-55].) The subleading contributions, even though they may be more important at a large $s$, are irrelevant for the current discussion since we are formally expanding the amplitude in terms of $1 / N$, where the unitarity should hold at each order.

Except for gauge boson exchange, it may well be the case that the saturation of the Froissart bound (up to $\log s$ ) and correspondingly the nonzero value of the large $|s|$ part of the integral are only possible by Pomeron exchange, which implies a universal growth of forward amplitudes irrespective of the quantum numbers. Assuming the universal asymptotic behavior of the amplitude, the authors of ref. [16] showed that the large $|s|$ contribution can be neglected for the scattering of real representations in theories with global symmetries by exploiting the crossing symmetry.

Our discussion seems to support the existence of the meta-stable vacuum in the $N_{f}=$ $N$ supersymmetric QCD. In order to establish the existence, however, one also needs to consider the double-trace terms, $\left(\operatorname{Tr}\left(M^{\dagger} M\right)\right)^{2}$, generated at $\mathcal{O}\left(1 / N^{2}\right)$ from the cylinder diagram as well as meson-baryon terms. The meson-baryon terms can be ignored once the $\mathrm{U}(1)$ baryon symmetry is gauged so that the baryons are heavy. Unfortunately, for the double-trace term, the imaginary part exists for both channels and also the large $s$ behavior is expected to be such that the large $|s|$ part does not vanish. It is difficult in this situation to determine the sign. One may ignore the double-trace term if the effects of the quark loops are parametrically suppressed so that the $\mathcal{O}\left(N_{f} / N\right)$ terms are counted as subleading contributions. This assumption may be supported (although not guaranteed) by the fact that the theory is in the confining phase rather than in the conformal window as well as by the success of the OZI rule [56-58] in the real QCD with $N_{f}=N=3$.

\section{$6 \quad$ String theory amplitudes}

We have discussed that $c$ is positive by considering the disk amplitude in the large $N$ theory. Because of the similarity between the large $N$ expansion of gauge theories and the $g_{\mathrm{s}}$ expansion of perturbative string theory, our discussion may be applicable also to the open-string sector of string theory. 
The quartic Kähler terms, which correspond to dimension-six four-fermion terms, are generated by disk amplitudes of the open strings in intersecting D-brane backgrounds. In models with $N$ D $p$-branes and $N^{\prime} \mathrm{D} p^{\prime}$-branes, $\mathrm{U}(N)$ and $\mathrm{U}\left(N^{\prime}\right)$ gauge fields arise from strings whose ends are on the $\mathrm{D} p$-branes and $\mathrm{D} p^{\prime}$-branes, respectively. We also have bifundamental matter $X$ from strings which end on a $\mathrm{D} p$-brane and a $\mathrm{D} p^{\prime}$-brane. Considering the scattering of the bifundamental matter $X X^{\dagger} \rightarrow X X^{\dagger}$, there are no $u$-channel poles nor cuts at the leading order of perturbation. This tells us that the amplitude has no imaginary parts along the real $s<0$ line for the forward scattering. On the other hand, we have an infinite tower of massive intermediate string states for the $s$-channel. In section 4 , we have seen that their contributions are positive when evaluating $c$.

For example, the four-fermion amplitude for the proton decay in ref. [59] can be matched to the quartic Kähler term at low energy. Since the sign is the same as that in the case of the massive gauge boson exchange, it corresponds to $c>0$. On the other hand, a similar analysis in ref. [60] for the contact four-fermion interactions reported that the contributions to the coefficients from the string oscillator modes turn out to have the opposite sign to the counterpart of QFT contributions, and thus $c<0$.

Both amplitudes are given in the form of

$$
A_{X X^{\dagger}}(s, t)=g_{\mathrm{s}} l_{\mathrm{s}}^{2}(s+t) \int_{0}^{1} d x x^{-1-l_{\mathrm{s}}^{2} s}(1-x)^{-1-l_{\mathrm{s}}^{2} t} G(x),
$$

where $g_{\mathrm{s}}$ and $l_{\mathrm{s}}$ are, respectively, the string coupling constant and the string length, and $G(x)$ represents model-dependent non-negative functions of $x$. In both of the examples, one can explicitly see that $A_{X X^{\dagger}}(s, 0) / s$ vanishes at a large $|s|$. In this situation, one should find $c>0$ as there is no discontinuity for $s<0$. By looking closely at the amplitude which gives $c<0$, we find that the sign of the residues of the string oscillator poles are opposite to those of gauge bosons, which seems to violate unitarity.

When $X$ is embedded as above (and if string theory is unitary), $c>0$ is quite general. In the actual applications such as for the inflation models, we are interested in the coefficients obtained by integrating out massive fields which are absent in the effective theory. In this case, the amplitude has the same structure as the large $N$ theory with $N_{f}$ fixed. By the same discussion around eq. (5.5), the large $|s|$ behavior should be good enough for a negative $t$, and one should be able to take the $t \rightarrow 0$ limit smoothly.

\section{Conclusion}

Unitarity is a fundamental requirement of the quantum theory, and is incorporated automatically in the definitions of the theory. However, when we consider the low energy effective theories, the implementation of the unitarity becomes quite non-trivial. The unitarity looks just violated at high energy. This actually turns out to be good news. By requiring that the UV theory is unitary, one can constrain the parameters in the effective theory.

For dimension-eight operators such as $\left(\partial_{\mu} \pi\right)^{4}$, the corresponding scattering amplitudes are $\mathcal{O}\left(s^{2}\right)$. In this case, it has been shown that the coefficients are positive by unitarity [4]. This is quite surprising when we are working within the effective theories. Both signs looked allowed in principle from the low energy perspective, at least in the trivial background. 
We extend the study to dimension-six operators such as $|\phi|^{2} \partial^{\mu} \phi^{\dagger} \partial_{\mu} \phi$. In this case, the sign of the coefficient is not universally determined while it is controlled by the relative sizes of the total cross sections of $\phi \phi^{\dagger}$ and $\phi \phi$ scatterings if the high energy behavior is good enough. In contrast to the case with the dimension-eight higher-derivative terms, the dimension-six term itself never shows the violation of the global notion of causality such as superluminality in low energy. When a particular class of UV theories is assumed, however, the sign of the dimension-six operator in the effective theory is restricted. We found that in supersymmetric gauge theories, the single-trace four-meson operator in the Kähler potential has a negative sign $\left(c>0\right.$, see eq. (2.1)) because there are vector resonances in the $M M^{\dagger}$ channel whereas no resonance in the $M M$ one. The analysis in this paper applies not only for four-scalar operators, but also for four-fermion operators, $\bar{\psi} \gamma^{\mu} P_{L} \psi \bar{\psi} \gamma_{\mu} P_{L} \psi$ and boson-fermion operators, $i \bar{\psi} \gamma^{\mu} P_{L} \psi \phi^{\dagger} \partial_{\mu} \phi+$ h.c. A similar discussion was given in ref. [6] for the scattering amplitudes of heavy fermions rather than the massless ones in which we are interested. See ref. [61] for the extension to the cases with an arbitrary spin. There have also been discussions on the sign of anomalous dimensions based on unitarity [62].

The dimension-six terms appear in various kinds of phenomena. For example, in the context of inflation, the negative Kähler terms are favored or disfavored depending on models. This means that the UV completion of each model is restricted in a certain direction. For example, the hybrid inflation model should not be UV completed to supersymmetric QCD-like theories or open string sectors in string theory.

It is sometimes the case that there are no possible intermediate states for $u$-channel processes, hence no poles nor cuts along the real negative $s$ for the forward scattering. In such a situation, $c>0$ once the large $|s|$ part of the contour vanishes. Similarly, if there are no poles nor cuts on the real positive $s$ for forward scattering, one can conclude $c<0$ once the large $|s|$ part vanishes. Therefore, we list up several situations in which we can establish that the large $|s|$ part of the integral vanishes. With the discontinuities only in either a positive or negative side of the real axis, the large $|s|$ integral can be ignored if any one of the following conditions is met:

- We can directly confirm that the amplitude is damped fast enough at high energy, i.e., $|A(s, 0) / s| \rightarrow 0$ as $|s| \rightarrow \infty$.

- There is a classical picture of scattering by a geometric cross section at high energy so that $\operatorname{Re} A(s, 0) \ll \operatorname{Im} A(s, 0)$ is ensured [63].

- The ratio $A(s, t) / s \rightarrow 0$ as $|s| \rightarrow \infty$ for a negative $t$, and there is no massless (nor tachyonic) state mediating $t$-channel processes.

The first case is trivial. We directly confirmed it in the case of the string amplitudes. The second criterion is met in QCD-like theories as discussed in the case of large $N$ theories with $N_{f} / N$ fixed. In the third case, we utilize the convergence in the $t<0$ region and extrapolate it to $t=0$. The extrapolation is justified in the absence of the $t=0$ pole in the scattering amplitude. This argument was used in the case of large $N$ theories with $N_{f}$ fixed.

The quartic term of the Kähler potential is essentially the Kähler curvature. Applying our discussion to the cases with the nonvanishing scalar background, $\langle X\rangle \neq 0$, we may 
infer the geometry of the Kähler manifold within the region whose size is at most the cutoff scale, $|X| \lesssim \Lambda$. It is interesting to note that parameters of the effective theory are further constrained non-trivially depending on the topology of the Kähler manifold. For example, the parameters of the supergravity models are quantized depending on the topology [64].

Finally we comment on higher dimensional operators. Although we focused on the dimension-six operators such as $|\phi|^{2} \partial^{\mu} \phi^{\dagger} \partial_{\mu} \phi$, the discussion is readily extended to operators which include higher derivatives. For instance, let us consider an operator constructed from four scalar fields and $2 m$ derivatives. In the low energy limit, the amplitude due to such an operator should behave as $\mathcal{O}\left(s^{m}\right)$, and hence we can consider

$$
\frac{1}{2 \pi i} \int_{C} d s \frac{A(s, 0)}{s^{m+1}}
$$

with the contour in figure 1 , to examine the sign of the coefficient. We studied $m=1$ in this paper, and $m=2$ (and also other even $m$ ) is discussed in ref. [4]. For $m \geq 2$, the discussion is greatly simplified since the large $|s|$ part always vanishes due to the Froissart bound. Thus for $m \geq 2$, the sign is always positive for an even $m$, while the relative size of $s$ - and $u$-channel processes determines the sign for an odd $m$.

\section{Acknowledgments}

This work is supported by JSPS KAKENHI Grant No. JP18J00540 (YE), JP15H03669 (RK), JP15KK0176 (RK), JP17J00731 (TT), MEXT KAKENHI Grant No. JP25105011 (RK), JP18H05542 (RK), and JSPS Research Fellowship for Young Scientists (YE, TT).

Open Access. This article is distributed under the terms of the Creative Commons Attribution License (CC-BY 4.0), which permits any use, distribution and reproduction in any medium, provided the original author(s) and source are credited.

\section{References}

[1] A. Martin, Unitarity and high-energy behavior of scattering amplitudes, Phys. Rev. 129 (1963) 1432 [INSPIRE].

[2] T.N. Pham and T.N. Truong, Evaluation of the Derivative Quartic Terms of the Meson Chiral Lagrangian From Forward Dispersion Relation, Phys. Rev. D 31 (1985) 3027 [INSPIRE].

[3] B. Ananthanarayan, D. Toublan and G. Wanders, Consistency of the chiral pion pion scattering amplitudes with axiomatic constraints, Phys. Rev. D 51 (1995) 1093 [hep-ph/9410302] [INSPIRE].

[4] A. Adams, N. Arkani-Hamed, S. Dubovsky, A. Nicolis and R. Rattazzi, Causality, analyticity and an IR obstruction to UV completion, JHEP 10 (2006) 014 [hep-th/0602178] [INSPIRE].

[5] A. Jenkins and D. O'Connell, The Story of O: Positivity constraints in effective field theories, hep-th/0609159 [INSPIRE].

[6] A. Adams, A. Jenkins and D. O'Connell, Signs of analyticity in fermion scattering, arXiv:0802.4081 [INSPIRE]. 
[7] G.R. Dvali, G. Gabadadze and M. Porrati, 4-D gravity on a brane in 5-D Minkowski space, Phys. Lett. B 485 (2000) 208 [hep-th/0005016] [INSPIRE].

[8] M. Dine, G. Festuccia and Z. Komargodski, A Bound on the Superpotential, JHEP 03 (2010) 011 [arXiv:0910.2527] [INSPIRE].

[9] Z. Komargodski and A. Schwimmer, On Renormalization Group Flows in Four Dimensions, JHEP 12 (2011) 099 [arXiv:1107.3987] [INSPIRE].

[10] L. O'Raifeartaigh, Spontaneous Symmetry Breaking for Chiral Scalar Superfields, Nucl. Phys. B 96 (1975) 331 [INSPIRE].

[11] S. Ray, Some properties of meta-stable supersymmetry-breaking vacua in Wess-Zumino models, Phys. Lett. B 642 (2006) 137 [hep-th/0607172] [InSPIRE].

[12] R. Kitano and Y. Ookouchi, Supersymmetry breaking and gauge mediation in models with a generic superpotential, Phys. Lett. B 675 (2009) 80 [arXiv:0812.0543] [InSPIRE].

[13] Z. Komargodski and D. Shih, Notes on SUSY and R-Symmetry Breaking in Wess-Zumino Models, JHEP 04 (2009) 093 [arXiv:0902.0030] [INSPIRE].

[14] I. Low, R. Rattazzi and A. Vichi, Theoretical Constraints on the Higgs Effective Couplings, JHEP 04 (2010) 126 [arXiv:0907.5413] [INSPIRE].

[15] A. Falkowski, S. Rychkov and A. Urbano, What if the Higgs couplings to $W$ and $Z$ bosons are larger than in the Standard Model?, JHEP 04 (2012) 073 [arXiv:1202.1532] [INSPIRE].

[16] B. Bellazzini, L. Martucci and R. Torre, Symmetries, Sum Rules and Constraints on Effective Field Theories, JHEP 09 (2014) 100 [arXiv:1405.2960] [INSPIRE].

[17] A.E. Nelson and N. Seiberg, $R$ symmetry breaking versus supersymmetry breaking, Nucl. Phys. B 416 (1994) 46 [hep-ph/9309299] [InSPIRE].

[18] K.A. Intriligator, N. Seiberg and D. Shih, Dynamical SUSY breaking in meta-stable vacua, JHEP 04 (2006) 021 [hep-th/0602239] [INSPIRE].

[19] A. Katz, Y. Shadmi and T. Volansky, Comments on the meta-stable vacuum in $N(f)=N(c)$ SQCD and direct mediation, JHEP 07 (2007) 020 [arXiv:0705.1074] [INSPIRE].

[20] A. Hetz and G.A. Palma, Sound Speed of Primordial Fluctuations in Supergravity Inflation, Phys. Rev. Lett. 117 (2016) 101301 [arXiv:1601.05457] [INSPIRE].

[21] G.R. Dvali, Q. Shafi and R.K. Schaefer, Large scale structure and supersymmetric inflation without fine tuning, Phys. Rev. Lett. 73 (1994) 1886 [hep-ph/9406319] [InSPIRE].

[22] Planck collaboration, Y. Akrami et al., Planck 2018 results. X. Constraints on inflation, arXiv: 1807.06211 [INSPIRE].

[23] M. Bastero-Gil, S.F. King and Q. Shafi, Supersymmetric Hybrid Inflation with Non-Minimal Kähler potential, Phys. Lett. B 651 (2007) 345 [hep-ph/0604198] [InSPIRE].

[24] K. Kumekawa, T. Moroi and T. Yanagida, Flat potential for inflaton with a discrete $R$ invariance in supergravity, Prog. Theor. Phys. 92 (1994) 437 [hep-ph/9405337] [INSPIRE].

[25] K.I. Izawa and T. Yanagida, Natural new inflation in broken supergravity, Phys. Lett. B 393 (1997) 331 [hep-ph/9608359] [INSPIRE].

[26] K.I. Izawa, Supergravity minimal inflation and its spectral index revisited, Phys. Lett. B 576 (2003) 1 [hep-ph/0305286] [INSPIRE]. 
[27] M. Kawasaki, M. Yamaguchi and T. Yanagida, Natural chaotic inflation in supergravity, Phys. Rev. Lett. 85 (2000) 3572 [hep-ph/0004243] [INSPIRE].

[28] L. Álvarez-Gaumé, C. Gomez and R. Jimenez, Minimal Inflation, Phys. Lett. B 690 (2010) 68 [arXiv: 1001.0010] [INSPIRE].

[29] L. Álvarez-Gaumé, C. Gomez and R. Jimenez, A Minimal Inflation Scenario, JCAP 03 (2011) 027 [arXiv: 1101.4948] [INSPIRE].

[30] A. Achucarro, S. Mooij, P. Ortiz and M. Postma, Sgoldstino inflation, JCAP 08 (2012) 013 [arXiv: 1203.1907] [INSPIRE].

[31] K.I. Izawa and Y. Shinbara, Supersymmetric tuned inflation, arXiv:0710.1141 [INSPIRE].

[32] S.V. Ketov and T. Terada, Inflation in supergravity with a single chiral superfield, Phys. Lett. B 736 (2014) 272 [arXiv:1406.0252] [INSPIRE].

[33] S.V. Ketov and T. Terada, Generic Scalar Potentials for Inflation in Supergravity with a Single Chiral Superfield, JHEP 12 (2014) 062 [arXiv: 1408.6524] [INSPIRE].

[34] S.V. Ketov and T. Terada, On SUSY Restoration in Single-Superfield Inflationary Models of Supergravity, Eur. Phys. J. C 76 (2016) 438 [arXiv: 1606.02817] [INSPIRE].

[35] R. Kallosh, A. Linde and D. Roest, Superconformal Inflationary $\alpha$-Attractors, JHEP 11 (2013) 198 [arXiv:1311.0472] [INSPIRE].

[36] D. Roest and M. Scalisi, Cosmological attractors from $\alpha$-scale supergravity, Phys. Rev. D 92 (2015) 043525 [arXiv: 1503.07909] [INSPIRE].

[37] A. Linde, Single-field $\alpha$-attractors, JCAP 05 (2015) 003 [arXiv:1504.00663] [INSPIRE].

[38] K. Nakayama, K. Saikawa, T. Terada and M. Yamaguchi, Structure of Kähler potential for D-term inflationary attractor models, JHEP 05 (2016) 067 [arXiv: 1603. 02557] [INSPIRE].

[39] L. Covi, M. Gomez-Reino, C. Gross, J. Louis, G.A. Palma and C.A. Scrucca, Constraints on modular inflation in supergravity and string theory, JHEP 08 (2008) 055 [arXiv:0805.3290] [INSPIRE].

[40] N. Seiberg, Exact results on the space of vacua of four-dimensional SUSY gauge theories, Phys. Rev. D 49 (1994) 6857 [hep-th/9402044] [INSPIRE].

[41] G. Veneziano, Some Aspects of a Unified Approach to Gauge, Dual and Gribov Theories, Nucl. Phys. B 117 (1976) 519 [inSPIRE].

[42] C. Csáki, M. Schmaltz and W. Skiba, Confinement in $N=1$ SUSY gauge theories and model building tools, Phys. Rev. D 55 (1997) 7840 [hep-th/9612207] [INSPIRE].

[43] I.I. Pomeranchuk, Equality of the Nucleon and Antinucleon Total Interaction Cross Section at High Energies, Sov. Phys. JETP 7 (1958) 499.

[44] S. Weinberg, Cross Sections at High Energies, Phys. Rev. 124 (1961) 2049 [INSPIRE].

[45] S. Caron-Huot, Z. Komargodski, A. Sever and A. Zhiboedov, Strings from Massive Higher Spins: The Asymptotic Uniqueness of the Veneziano Amplitude, JHEP 10 (2017) 026 [arXiv: 1607.04253] [INSPIRE].

[46] S.J. Brodsky and G.R. Farrar, Scaling Laws at Large Transverse Momentum, Phys. Rev. Lett. 31 (1973) 1153 [INSPIRE].

[47] G.P. Lepage and S.J. Brodsky, Exclusive Processes in Perturbative Quantum Chromodynamics, Phys. Rev. D 22 (1980) 2157 [InSPIRE]. 
[48] O. Andreev and W. Siegel, Quantized tension: Stringy amplitudes with Regge poles and parton behavior, Phys. Rev. D 71 (2005) 086001 [hep-th/0410131] [INSPIRE].

[49] G. Veneziano, S. Yankielowicz and E. Onofri, A model for pion-pion scattering in large- $N$ QCD, JHEP 04 (2017) 151 [arXiv:1701.06315] [INSPIRE].

[50] M. Froissart, Asymptotic behavior and subtractions in the Mandelstam representation, Phys. Rev. 123 (1961) 1053 [INSPIRE].

[51] I.I. Pomeranchuk, The Conservation of Isotopic Spin and the Scattering of Antinucleons by Nucleons, Sov. Phys. JETP 3 (1956) 306.

[52] B. Okun and I.I. Pomeranchuk, The Conservation of Isotopic Spin and the Cross Section of the Interaction of High-Energy $\pi$-Mesons and Nucleons with Nucleons, Sov. Phys. JETP 3 (1956) 307.

[53] L.L. Foldy and R.F. Peierls, Isotopic Spin of Exchanged Systems, Phys. Rev. 130 (1963) 1585 [INSPIRE].

[54] A.B. Kaidalov, High-energy hadronic interactions (20 years of the quark gluon strings model), Phys. Atom. Nucl. 66 (2003) 1994 [INSPIRE].

[55] C. Ewerz, The Odderon in quantum chromodynamics, hep-ph/0306137 [INSPIRE].

[56] S. Okubo, Phi meson and unitary symmetry model, Phys. Lett. 5 (1963) 165 [INSPIRE].

[57] G. Zweig, An SU(3) model for strong interaction symmetry and its breaking. Version 2, in Developments in the quark theory of hadrons. Vol. 1. 1964-1978, D. Lichtenberg and S.P. Rosen eds., pp. 22-101 (1964) [INSPIRE].

[58] J. Iizuka, Systematics and phenomenology of meson family, Prog. Theor. Phys. Suppl. 37 (1966) 21 [INSPIRE].

[59] I.R. Klebanov and E. Witten, Proton decay in intersecting D-brane models, Nucl. Phys. B 664 (2003) 3 [hep-th/0304079] [INSPIRE].

[60] I. Antoniadis, K. Benakli and A. Laugier, Contact interactions in D-brane models, JHEP 05 (2001) 044 [hep-th/0011281] [INSPIRE].

[61] B. Bellazzini, Softness and amplitudes' positivity for spinning particles, JHEP 02 (2017) 034 [arXiv: 1605.06111] [INSPIRE].

[62] K. Higashijima and E. Itou, Unitarity bound of the wave function renormalization constant, Prog. Theor. Phys. 110 (2003) 107 [hep-th/0304047] [INSPIRE].

[63] S. Weinberg, The Quantum theory of fields. Vol. 1: Foundations, Cambridge University Press (2005) [INSPIRE].

[64] E. Witten and J. Bagger, Quantization of Newton's Constant in Certain Supergravity Theories, Phys. Lett. B 115 (1982) 202 [InSPIRE]. 